\title{
Numerical Simulation of an Over-Expanded Supersonic Nozzle Flow
}

\author{
Faisal Al Qurooni ${ }^{1}$, Ali Vakil ${ }^{1,2}$, Ehab Elsaadawy ${ }^{3}$, Sheldon I. Green ${ }^{1}$ \\ ${ }^{I}$ Department of Mechanical Engineering, The University of British Columbia - Vancouver \\ 6250 Applied Science Ln, Vancouver, BC, Canada V6T 1Z4 \\ ${ }^{2}$ Coanda Research and Development Corporation \\ 6741 Cariboo Rd, Burnaby, BC, Canada, V3N $4 A 3$ \\ ${ }^{3}$ Saudi Aramco, Research \& Development Center, Dhahran 31311, KSA \\ Email:faisal90@alumni.ubc.ca (F.Al Qurooni),alivakil@mech.ubc.ca (A.Vakil), \\ ehab.elsaadawy@aramco.com (E.Elsaadawy),Sheldon.Green@ubc.ca (S.I.Green)
}

\begin{abstract}
Flaring in oil and gas production is the controlled burning of unwanted exhaust gases to enhance safety. Obtaining complete combustion and thus eliminating or reducing smoke is critical to meet increasingly strict environmental regulations. To improve flare combustion, gas flares are equipped with air nozzles that introduce extra oxygen and improve mixing in the combustion zone. These nozzles are operated in the subsonic, sonic, or supersonic regimes. In this paper we are concerned with turbulence modeling of the jet flow exiting from a particular convergent-divergent nozzle used in flare systems. The Realizable $k-\varepsilon$ and SST $k-\omega$ models are used to study the compressible flow within that specific nozzle, which has an exit diameter of $3.38 \mathrm{~mm}$ and has convergent and divergent sections that are connected via a throat section with a finite length and constant diameter. The velocity profiles and turbulent kinetic energy predicted by both turbulence models, in the vicinity of the nozzle outlet and along the symmetry axis of the nozzle, are compared for nozzle pressure ratios in the range $1.18 \leq \mathrm{NPR} \leq 1.78$. It is shown that for $M a \leq 1$, both turbulence models predict nearly identical flow evolution along the nozzle. When the flow becomes supersonic, the shock surface, and consequently nozzle outlet velocity profiles, predicted by the SST $k-\omega$ model deviates slightly from the other model. The differences, however, become negligible a couple of diameters downstream of the nozzle outlet. Computed entrainment rate coefficients vary slightly when changing the turbulence model, and this difference remains insignificant with increasing downstream distance.
\end{abstract}

Keywords: CD nozzle flow, compressible flow, turbulence models, entrainment rate, gas flaring

\section{INTRODUCTION}

Gas flaring is a technique used in several industries such as gas and oil separation plants (GOSPs), oil refineries, gas plants and other petrochemical plants in which unwanted or excess gases are burnt safely. Gas flares are also used to protect the plants against the danger of over pressurizing in case of emergency or critical equipment trip. The gas flaring system is very important and plants operations should cease if it is not working properly. While gas flaring is required for safety reasons, it has many drawbacks, including negative impacts on the environment (primarily due to incomplete combustion of the gas) and the waste of potentially useful resources. To mitigate these negative impacts, companies seek to eliminate or reduce gas flaring. In situations when flaring is unavoidable, it is desirable to have smokeless gas flaring (Figure 1). Saudi Aramco, the largest oil company in the world, has made a thorough analysis and study [1] to investigate a cost effective and efficient solution to retrofit its hundreds of gas flares in Saudi Arabia. Several options were explored such as Steam-Assisted Flare tips, sonic flare tips and LowPressure Air-Assist. However, none of these solutions was deemed to be cost effective and, therefore Saudi Aramco developed a new flare design named the High Pressure Air Assist System (HPAAS). Briefly, the system consists of convergent-divergent nozzles that surround the circumference of the flare exit tip. As these nozzles inject compressed air to the combustion zone, the resulting air entrainment from the atmosphere due to the high exit velocity provides the required air mass to yield smokeless flaring (Figure 1). 
For a given nozzle shape, the flow controlling parameter is the nozzle pressure ratio (NPR). In converging nozzles $(\mathrm{CN})$, as the NPR increases, the maximum flow rate is achieved when the flow speed at the nozzle exit is sonic, corresponding to the choked flow condition. A converging-diverging nozzle geometry (CDN) allows the flow to achieve supersonic speeds. For a CDN that undergoes an isentropic expansion the sonic condition is reached at the throat connecting an upstream subsonic condition to a downstream supersonic condition. In contrast, an isentropic compression within the nozzle connects an upstream supersonic condition to a downstream subsonic condition via a sonic throat [2]. If the flow is choked at the throat but the NPR differs from that required to yield isentropic flow, a combination of normal and oblique shocks is formed downstream of the throat [3]. Numerical predictions of the compressible flow within $\mathrm{CDN}$, shocks behavior, and the compressible turbulent shear flow of supersonic jets has been subject of extensive studies in the last century.

Eggers [4] conducted an analytical and experimental investigation of a supersonic jet (Mach 2.22 nozzle) to understand turbulence quantities and jet entrainment. He concluded that an eddy viscosity formulation independent of the radial coordinate, employed by other researchers, for a compressible turbulent jet was not justified. Birch and Eggers [5] collated experimental data on developed free turbulent shear layers to facilitate the validation of the turbulence models and numerical predictions. They concluded that the available data were not sufficient to clearly establish the effect of density gradients on mixing rate. Lau et al. [6] conducted measurements of a $51 \mathrm{~mm}$ diameter turbulent jet at Mach 0.28, 0.90, and 1.37 using a LDV and a hot-wire anemometer. Their data indicate a decrease in the spreading rate of the mixing layer with increasing Mach number. Seiner et al. [7] investigated the effect of jet temperature on the mixing rate, and concluded that high temperatures resulted in enhanced density fluctuations to increase the mixing rate.

Reynolds-averaged Navier-Stokes (RANS) solvers have been used by many researchers to simulate supersonic exhausts. A comprehensive review of various turbulence modelling, advantages and limitations of each approach is presented in [8]. Koch conducted a study on subsonic axisymmetric jets with flow separation using a two-equation $k-\varepsilon$ turbulence model [9]. While the mean flow values showed a good agreement with experiment, the mixing rate predicted by the numerical modelling was lower than the experimental results, and so were the turbulent kinetic energy levels. To incorporate compressibility effects in turbulence models, and consequently to better predict the decrease in the growth rate with increasing Mach number, an additional compressibility term should be included in the turbulence transport equations [10, 11]. Georgiadis et al. [12, 13] assessed the accuracy of modified two-equation turbulence models in flow field predictions of a subsonic (Mach number 0.5 ) heated and unheated jet. They showed that all the modified equations provided improved predictions compared to the standard models. However, all the models underestimated initial jet mixing rate and the turbulence kinetic energy fields.

Xiao et al. [14] examined experimentally and numerically the effect of both the nozzle expansion ratio and the NPR on the entrainment from CDN. The computations showed that the nozzle expansion ratio has a greater effect on the entrainment than the NPR. Among the RANS models, the SST $k-\omega$ model compared the best with experiment, which was attributed to its ability to predict the flow separation after the shock correctly. For a fixed nozzle expansion ratio of 1.5, their experimental study showed the best mixing was achieved when the NPR was in the range of 1.4-1.6. In a numerical study conducted on CDN with Mach number in the range $0.9<M a<1.2$ and NPR of 4 and 6, DalBello et al. [15] found that the SST $k-\omega$ model could match well with experimental measurements. The RANS models prediction accuracy decreases as the flow compressibility effects become more important. To account for these effects, modified RANS models were considered and their prediction performance were compared against experiment [16] [13].

Sarkar and Sarkar et al. [17, 18] studied compressible shear layers using a Reynolds Stress Model (RSM). When the compressibility effects were not considered explicitly, the model failed to predict the dependence of the shear layer growth rate on the Mach number. In comparison, when the compressibility effects were considered explicitly, the computed results compared better with experiment. Lijo et al. [19] used RSM to study transient flows in a rocket propulsion nozzle which involves free and restricted shock separation, and they found RSM model results were in good agreement with experiment. Balabel et al. [20] assessed RSM and different RANS models for gas flow in a two-dimensional CDN with steady, compressible and turbulent flow. In their investigation to predict the separation point and the shock wave location, they found that the SST $k-\omega$ model gave the best results when compared to experiment. The objective of this paper is to study the compressible turbulent flow picture within a specific converging- 
diverging nozzle with a finite length throat of constant area, and specifically to compare the nozzle flow field including the axial velocity, mass entrainment, Mach number and turbulent kinetic energy, predicted by different turbulence models.

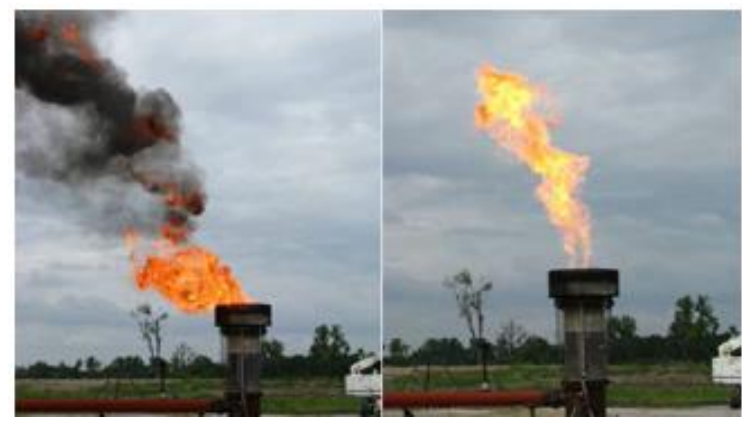

Figure 1: A flare with (right) and without (left) smokeless air, which shows the nozzles' advantage [1].

\section{NUMERICAL METHOD}

In this section, the numerical formulations employed to obtain the CFD results using ANSYS Fluent 18.0 are presented.

\subsection{Governing Equations}

The governing-equations require special treatment, i.e. closure, when the flow is turbulent. In the timeaveraging of the governing equations, the physical quantity $\phi$ is decomposed into its mean component $\bar{\phi}$ and fluctuating component $\phi^{\prime}$ via the Reynolds decomposition as $\phi=\bar{\phi}+\phi^{\prime}$. To account for the effects of the density fluctuations due to turbulence, Favre-averaging or density-weighed averaging is adopted in turbulent compressible flows. The densityweighted average of $\phi$ is obtained as $\phi=\tilde{\phi}+\phi^{\prime \prime}$, where $\tilde{\phi}=\overline{\rho \phi} / \bar{\rho}$. The details of the Favre averaging can be found in [21].

The mass-averaged conservation of mass in terms of mean velocity vector $\tilde{u}_{i}$ is given by Equation (1). The governing equations for the transport of momentum is given by Equation (2). $p$ is the mean pressure and related to mean temperature and density as $p=\bar{\rho} R \tilde{T}$. The viscous stress tensor $\tau_{i j}$ for an isentropic Newtonian fluid is defined as given by Equation (3) where $\delta_{i j}$ is the Kronecker delta function, and $\mu$ is the dynamic viscosity. The Reynolds stresses $\bar{R}_{i j}=$ $-\bar{\rho} \widetilde{u_{\imath}^{\prime \prime} u_{\jmath}^{\prime \prime}}$, arising from the Favre-averaging of the momentum equations, require modelling. One of the most common approaches to close the set of equations is to use the Boussinesq hypothesis to relate the Reynolds stresses to the mean deformation rates and the turbulent kinetic energy $k$ as given in Equation (4). The turbulent viscosity $\mu_{t}$ is given by $\mu_{t}=\rho C_{\mu} k^{2} / \varepsilon$, where $\varepsilon$ is the dissipation of turbulent kinetic energy and $C_{\mu}$ is a constant.

The Realizable $k-\varepsilon$ employs Equation (5) and Equation (6) as transport equations for $k$ and $\varepsilon$, respectively. $\bar{R}_{i j} \partial \tilde{u}_{i} / \partial x_{j}$ is the generation of turbulence kinetic energy due to the mean velocity gradients. $Y_{M}$ is the contribution of fluctuating dilation to the dissipation rate. $S=\sqrt{2 S_{i j} S_{i j}}$ is the modulus of the mean deformation rate tensor. $\sigma_{k}=1.0, \sigma_{\varepsilon}=$ 1.2 are, respectively, the turbulent Prandtl numbers for $k$ and $\varepsilon$. In contrast to the standard $k-\varepsilon$ model, $C_{\mu}$ is no longer a constant but rather depends on the mean deformation and rotation rates, the angular velocity of the rotation frame, and the turbulence quantities [22].

The SST $k-\omega$ employs Equations (8) and (9), respectively, as transport equations for $k$ and the specific dissipation rate $\omega . \sigma_{k}$ and $\sigma_{\omega}$ are the turbulent Prandtl numbers for $k$ and $\omega$, respectively. The turbulent viscosity is computed as $\mu_{t}=f \rho k / \omega$, where $f$ is a function of the strain rate magnitude, the specific dissipation rate, a limiter function, and a Reynolds number defined as $R e_{t}=\rho k / \mu \omega$. The details of the model constants can be found in [23]. This formulation accounts for the transport of the turbulence shear stress in the definition of the turbulent viscosity. The Favre-averaged energy equation is given by Equation (10). The turbulent flux $-\overline{\rho u_{\jmath}^{\prime \prime} h}$ is given based on the averaged enthalpy, turbulent Prandtl number, and the turbulent eddy viscosity as given in Equation (11). The Favreaveraged material derivative is defined in Equation (12).

\subsection{Meshing and Boundary Conditions}

Figure 2 shows the geometry of the specific nozzle studied in this paper. This geometry is used commonly in gas flares. The converging and diverging sections of this nozzle are connected via a throat with a constant diameter and a finite length. Figure 2 also shows the computational domain used for the simulations with the associated boundary conditions. The domain extends from $50 r_{\text {exit }}$ downstream of the nozzle exit, where $r_{\text {exit }}$ is the nozzle exit radius. The domain dimensions are given in Figure 2. The lateral walls, 
Table 1: List of Governing Equations

Conservation

of Mass

$$
\frac{\partial \bar{\rho}}{\partial t}+\frac{\partial}{\partial x_{i}}\left(\bar{\rho} \tilde{u}_{i}\right)=0
$$

$$
\frac{\partial}{\partial t}\left(\bar{\rho} \tilde{u}_{i}\right)+\frac{\partial}{\partial x_{j}}\left(\bar{\rho} \tilde{u}_{j} \tilde{u}_{i}\right)=-\frac{\partial p}{\partial x_{i}}+\frac{\partial}{\partial x_{j}}\left(\bar{\tau}_{i j}-\bar{R}_{i j}\right)
$$

Conversation

of

$$
\bar{\tau}_{i j}=\mu\left(\frac{\partial \tilde{u}_{i}}{\partial x_{j}}+\frac{\partial \tilde{u}_{j}}{\partial x_{i}}-\frac{2}{3} \frac{\partial \tilde{u}_{k}}{\partial x_{k}} \delta_{i j}\right)
$$

Momentum

Realizable

$$
\bar{R}_{i j}=-\bar{\rho} \widetilde{u_{\imath}^{\prime \prime} u_{\jmath}^{\prime \prime}}=\mu_{t}\left(\frac{\partial \tilde{u}_{i}}{\partial x_{j}}+\frac{\partial \tilde{u}_{j}}{\partial x_{i}}\right)-\left(\frac{2}{3} \rho k+\mu_{t} \frac{\partial \tilde{u}_{k}}{\partial x_{k}}\right) \delta_{i j}
$$

$$
\begin{gathered}
\frac{\partial}{\partial t}(\bar{\rho} k)+\frac{\partial}{\partial x_{j}}\left(\bar{\rho} \tilde{u}_{j} k\right)=\frac{\partial}{\partial x_{j}}\left(\left[\mu+\frac{\mu_{t}}{\sigma_{k}}\right] \frac{\partial k}{\partial x_{j}}\right)+\bar{R}_{i j} \frac{\partial \widetilde{u}_{i}}{\partial x_{j}}-\rho \varepsilon-Y_{M} \\
\frac{\partial}{\partial t}(\bar{\rho} \varepsilon)+\frac{\partial}{\partial x_{j}}\left(\bar{\rho} \tilde{u}_{j} \varepsilon\right)=\frac{\partial}{\partial x_{j}}\left(\left[\mu+\frac{\mu_{t}}{\sigma_{\varepsilon}}\right] \frac{\partial \varepsilon}{\partial x_{j}}\right)+\rho C_{1} S \varepsilon-\rho C_{2} \frac{\varepsilon^{2}}{k+\sqrt{v \varepsilon}}
\end{gathered}
$$$$
C_{1}=\max \left[0.43, \frac{\eta}{\eta+5}\right], \eta=S \frac{k}{\varepsilon}, C_{2}=1.9
$$$$
\mu_{t}=\rho C_{\mu} k^{2} / \varepsilon
$$

$k-\varepsilon$

$$
\frac{\partial}{\partial t}(\bar{\rho} k)+\frac{\partial}{\partial x_{j}}\left(\bar{\rho} \tilde{u}_{j} k\right)=\frac{\partial}{\partial x_{j}}\left(\left[\mu+\frac{\mu_{t}}{\sigma_{k}}\right] \frac{\partial k}{\partial x_{j}}\right)+\bar{R}_{i j} \frac{\partial \widetilde{u}_{i}}{\partial x_{j}}-\rho \beta^{*} k \omega
$$

SST $k-\omega$

$$
\begin{aligned}
& \frac{\partial}{\partial t}(\bar{\rho} \omega)+\frac{\partial}{\partial x_{j}}\left(\bar{\rho} \tilde{u}_{j} \omega\right)=\frac{\partial}{\partial x_{j}}\left(\left[\mu+\frac{\mu_{t}}{\sigma_{\omega}}\right] \frac{\partial \omega}{\partial x_{j}}\right)+\frac{\alpha \alpha^{*}}{v_{t}} \bar{R}_{i j} \frac{\partial \widetilde{u}_{i}}{\partial x_{j}}-\rho \beta \omega^{2}+\gamma \rho \frac{1}{\omega \sigma_{w, 2}} \frac{\partial k}{\partial x_{j}} \frac{\partial \omega}{\partial x_{j}} \\
& \mu_{t}=f \rho k / \omega \\
& \frac{\partial}{\partial t}(\bar{\rho} \tilde{h})+\frac{\partial}{\partial x_{j}}\left(\bar{\rho} \tilde{u}_{j} \tilde{h}\right)=\frac{\widetilde{D} \bar{p}}{\partial t}+\overline{u_{\jmath}^{\prime \prime} \frac{\partial p}{\partial x_{J}}}+\frac{\partial}{\partial x_{j}}\left(-\overline{\rho u_{\jmath}^{\prime \prime} h}\right)+\bar{\tau}_{i j} \frac{\partial \tilde{u}_{j}}{\partial x_{j}}+\overline{\tau_{l \jmath} \frac{\partial u_{J}^{\prime \prime}}{\partial x_{J}}}+\frac{\partial}{\partial x_{j}}\left(\frac{\mu}{P r} \frac{\partial \tilde{h}}{\partial x_{j}}\right)
\end{aligned}
$$

Conservation of Energy

$$
\begin{gathered}
-\overline{\rho u_{\jmath}^{\prime \prime h}}=\frac{\mu_{t}}{P r_{t}} \frac{\partial \tilde{h}}{\partial x_{j}} \\
\frac{\widetilde{D}}{D t}=\frac{\partial}{\partial t}+\tilde{u}_{j} \frac{\partial}{\partial x_{j}}
\end{gathered}
$$
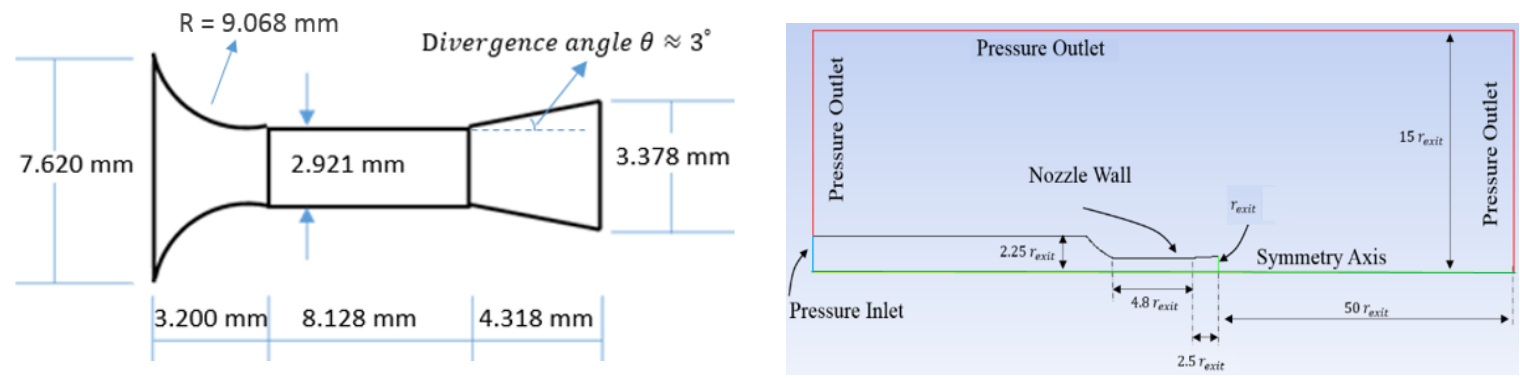

Figure 2: (Left) The geometry of the nozzle. (Right) Schematic of the computational domain used for the simulations with the associated boundary conditions; the dimensions of the computational domain are also shown in terms of nozzle exit radius $r_{\text {exit }}$ (not shown to scale). 
considered as the pressure outlet boundary condition, are extended to $15 r_{\text {exit }}$ to assure their impact on the jet flow is insignificant. The typical computational mesh used in the simulations, shown in Figure 3, highlights the small mesh size used in regions of high velocity gradients and around the shock location.

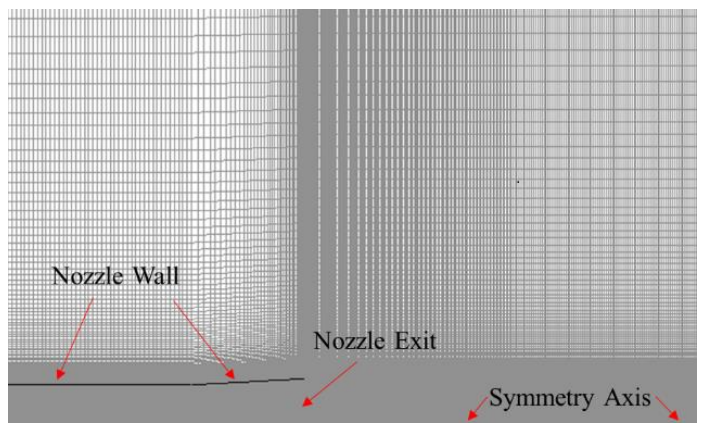

Figure 3: The typical computational mesh used in the simulation; the mesh around the nozzle exit is only shown for the sake of illustration.

\subsection{Mesh Independency Check}

To ensure that the simulations are mesh-independent, several flows were simulated with different mesh densities (number of cells was $1 \times 10^{5}, 1.5 \times 10^{5}$ and $3 \times 10^{5}$ for the coarse, medium and fine mesh, respectively). In the refinement procedure, we refined the boundary layer mesh close to the nozzle wall, the flow region around the jet shear layer, and the shock areas. Additionally, we slightly refined the mesh in the outer region of the flow. The velocity profiles at $\mathrm{x}=2.256 r_{\text {exit }}$ downstream of the nozzle exit for $\mathrm{P}_{\text {in }}=$ $160 \mathrm{kPaa}$ for different mesh sizes are shown in Figure 4. To estimate the discretization error, we followed the procedure recommended in [24] for the velocity profiles. The numerical uncertainty in the fine-grid solution was found to be $1.6 \%$ for the velocity profiles.

\subsection{D vs. 3D Comparison}

For a highly over-expanded flow within a converging -diverging nozzle, the flow is inherently not axisymmetric [25]. The complex pattern of the shock surfaces (oblique and normal shocks) is threedimensional, which means that the azimuthal derivatives of quantities should be accounted for in the governing equations. However, for the range of operating conditions of interest in this study, the flow remains attached to the nozzle walls and no flow separation was observed. These characteristics should permit one to use with reasonable accuracy a $2 \mathrm{D}$ axisymmetric model instead of full three-dimensional modelling. Using the 2D model avoids long computational times.

To verify that the $2 \mathrm{D}$ axisymmetric modelling is capable of capturing the shock surfaces and flow details, some 3D simulations were conducted to compare the simulations results with those of the corresponding $2 \mathrm{D}$ axisymmetric simulations. The SST $k-\omega$ turbulence model was chosen for both sets of simulations. The Mach number and the pressure coefficient profiles along the nozzle symmetry axis for the same upstream pressure are shown in Figure 5. The 2D profiles are almost identical to their 3D counterparts.

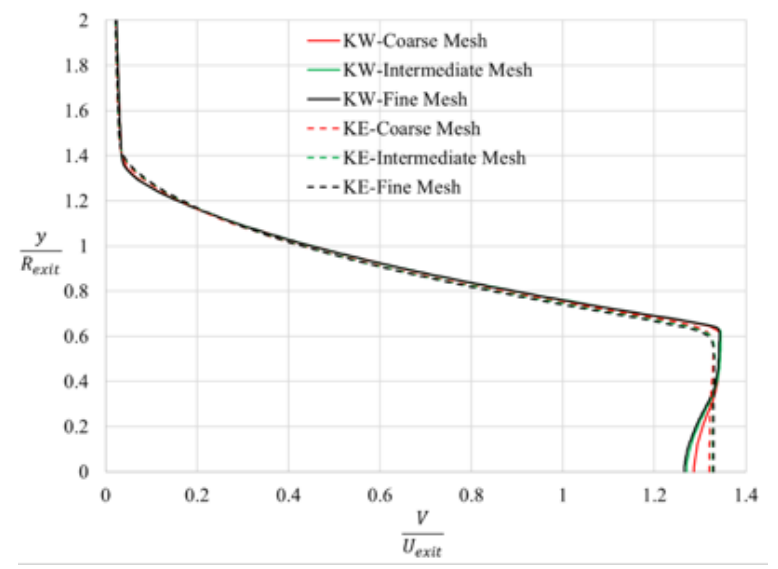

Figure 4: Velocity profile at $\mathrm{x}=2.256 \mathrm{r}_{\text {exit }}$ downstream of the nozzle exit for $P_{i n}=160 \mathrm{kPaa}$.

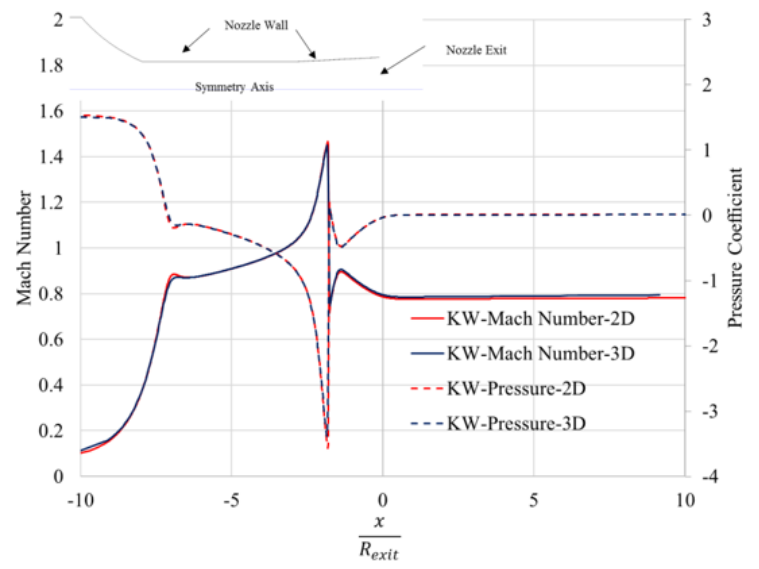

Figure 5: Mach number and pressure coefficient profiles along the nozzle symmetry axis for 2D axisymmetric and 3D simulation. The upstream pressure is $\mathrm{P}_{\mathrm{in}}=160 \mathrm{kPaa}$. 


\subsection{Model Validation against Experimental Data}

This section contains a comparison between the simulation results on the Acoustic Reference Nozzle (ARN) [26] from the ANSYS Fluent solver and those reported in [13] and [26]. The geometry details are reported in [26]. The jet is issued from a nozzle with an exit diameter of $50.8 \mathrm{~mm}$. The Setpoint 3, where the jet is unheated $\left(\mathrm{T}_{\mathrm{j}} / \mathrm{T}_{\infty}=0.950\right)$ and the compressibility effects $(\mathrm{Mach}=0.513)$ on the flow evolution could be insignificant, was chosen for the comparison reported here. The NPR of this point is 1.197 .

Figure 6 and Figure 7, respectively, show a comparison of the axial velocity and turbulent kinetic energy along the symmetry axis between the current simulations and those reported in [13] and [26]. In Figure 6, the present simulation shows a good agreement with both the experimental data and previous simulation [13] results.

Both the present simulation and the previous simulation [13] predict a longer potential core than that observed experimentally. The current simulation, however, predicts a potential core shorter than the previous simulation [13]. Furthermore, the current simulation predicts a decay rate faster than both the experimental results and the previous simulation [13]. On the other hand, the turbulent kinetic energy (TKE) predicted by the current simulation using the SST $k-$ $\omega$ turbulence model is in better agreement with experimental data than the previous simulations [13].

A comparison of the velocity profile at different positions downstream of the nozzle exit is shown in Figure 8. Very good agreement exists in the dimensions of the potential core and shear layer between the experimental data, the earlier simulation [13], and the current simulation. The above results show that the utilized modeling approach in this paper is adequate to capture the flow features of the flow field studies in this investigation, especially at Mach number of 0.5 .

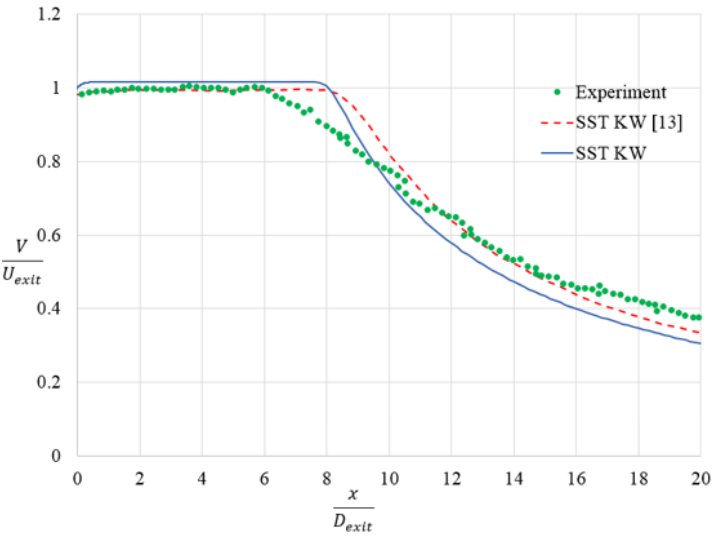

Figure 6: Comparison of axial velocity profile along the symmetry axis between current simulations, those in [13], and experiments [26].

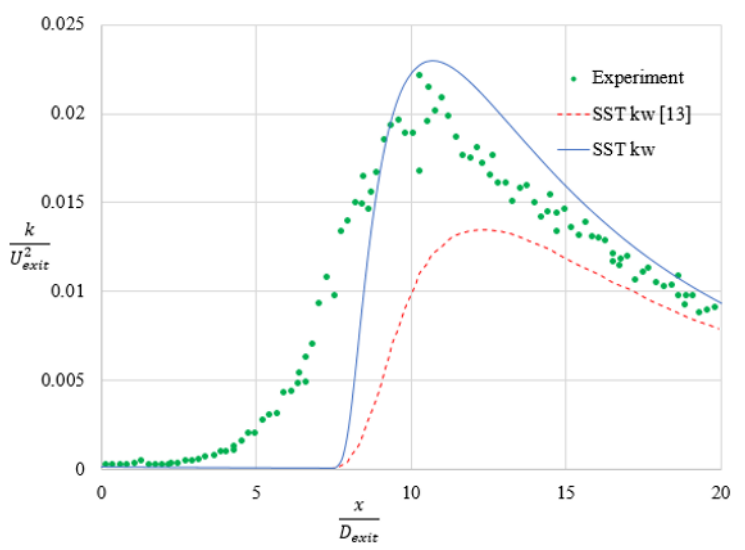

Figure 7: Comparison of turbulent kinetic energy profile along the axis of symmetry between current simulations, those in [13], and experiments [26].

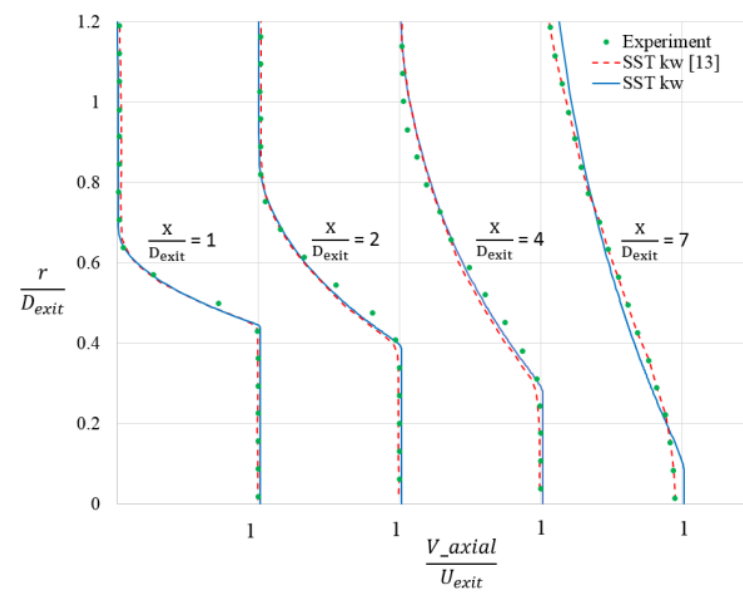

Figure 8: Comparison of velocity profile downstream of the nozzle exit between current simulations and results in [13] and [26]. 


\section{RESULTS AND DISCUSSIONS}

In this section, simulation results of the convergingconstant throat-diverging nozzle, as shown in Figure 2 , are presented. The nozzle has an exit to throat area ratio of 1.34. The flow fields are studied at different pressure ratios (NPR) in the range $1.18 \leq \mathrm{NPR} \leq$ 1.78 , corresponding to $120 \mathrm{kPa} \leq \mathrm{P}_{\text {in }} \leq 180 \mathrm{kPa}$. The downstream pressure is set to standard atmospheric pressure at sea level $(101 \mathrm{kPa})$. The working fluid is air. Velocity, TKE, and Mach number variations within and downstream of the nozzle are presented.

\subsection{Mean Axial Velocity Profiles}

Figure 9 shows a comparison of the normalized axial velocity profiles as computed by the different turbulence models for two upstream pressures: $\mathrm{P}_{\mathrm{in}}=$ $140 \mathrm{kPaa}$ and $\mathrm{P}_{\mathrm{in}}=160 \mathrm{kPaa}$. The profiles are extracted at two locations downstream of the nozzle exit, $\mathrm{x}_{1}=2.256 \mathrm{r}_{\text {exit }}$ and $\mathrm{x}_{2}=11.278 \mathrm{r}_{\text {exit }}$.

The normalized velocity profiles predicted by different turbulence models follow each other closely at $\mathrm{P}_{\text {in }}=140 \mathrm{kPaa}$; they start with a potential core close to the jet centerline and transition to the slow moving outer flow through a shear layer. The potential core at $\mathrm{x}=2.256 \mathrm{r}_{\text {exit }}$ exists in $0<\mathrm{y} / \mathrm{r}_{\text {exit }}<0.6$. The extent of the potential core in the cross-stream direction at $\mathrm{x}=11.278 \mathrm{r}_{\text {exit }}$ shrinks to $0<\mathrm{y} / \mathrm{r}_{\text {exit }}<$ 0.1 due to the mixing. The SST $\mathrm{k}-\omega$ model predicts a slightly faster decay in the potential core compared to the Realizable $\mathrm{k}-\varepsilon$ model. The shear layer is contained in $0.6<y / \mathrm{r}_{\text {exit }}<1.5$ at $\mathrm{x}=2.256 \mathrm{r}_{\text {exit }}$, while it has a far-reaching extent $0.1<\mathrm{y} / \mathrm{r}_{\text {exit }}<$ 3.5 at $\mathrm{x}=11.278 \mathrm{r}_{\text {exit }}$ (for comparison purposes the profiles at $\mathrm{x}=11.278 \mathrm{r}_{\text {exit }}$ are clipped to $\mathrm{y} / \mathrm{r}_{\text {exit }}=$ 2.0). The flow at this inlet pressure approaches sonic conditions at the throat.

At $\mathrm{P}_{\text {in }}=160 \mathrm{kPaa}$ the velocity profiles predicted by the Realizable $\mathrm{k}-\varepsilon$ model are similar to those at the lower pressure except that the potential core reaches a higher normalized peak velocity. The SST $k-\omega$, however, predicts a velocity deficit in the near field in the potential core, which seems unphysical. This can be seen at the centerline $(\mathrm{y}=0)$ as velocity profile is dented backward between $0<y / r_{\text {exit }}<0.4$. Note that the flow within the nozzle at this pressure is supersonic (both turbulence models predict a peak Mach number of $\approx 1.45$ in the diverging section), where the compressibility effects play a critical role in the flow evolution. This velocity deficit, however, disappears in the far field (after $\mathrm{x} \geq 11.278 \mathrm{r}_{\text {exit }}$ ). In fact, both turbulence models predict almost the same normalized axial velocity profile in the far field at $\mathrm{x}=11.278 \mathrm{r}_{\text {exit }}$ regardless of the flow condition.
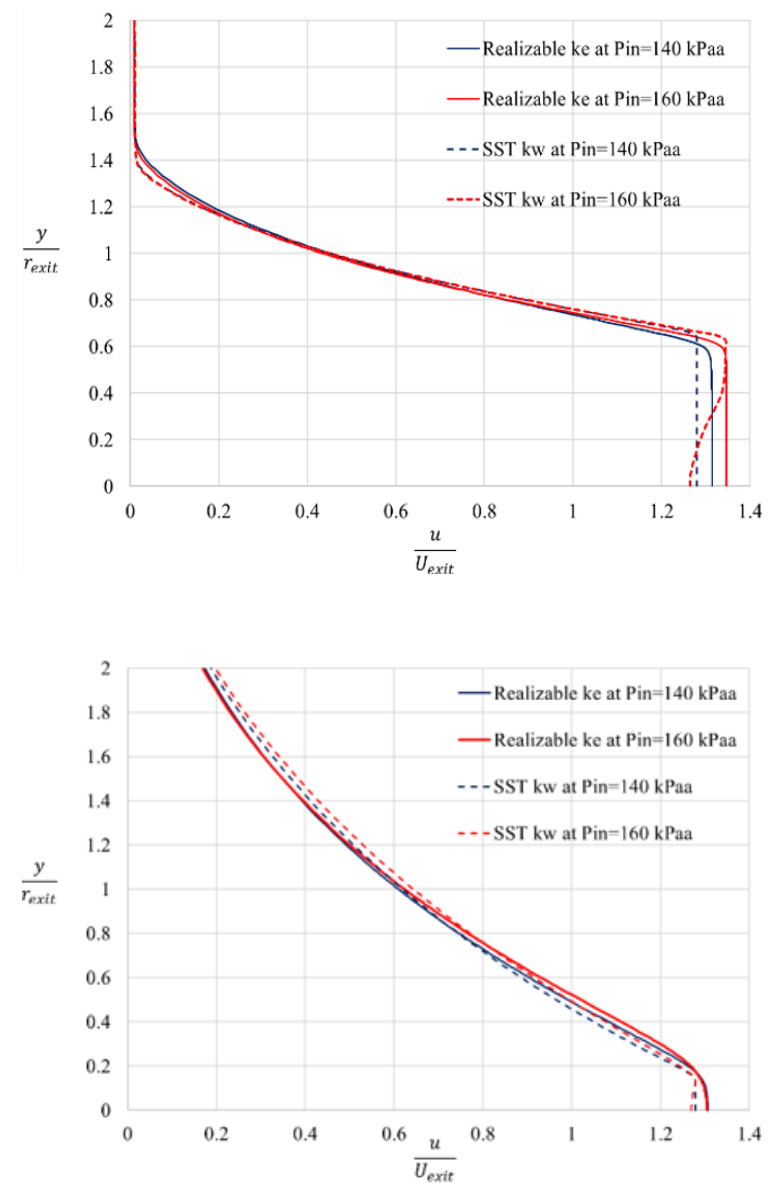

Figure 9: The normalized axial velocity profiles downstream of the nozzle exit for the different turbulence models; Extraction line is at (top) $\mathrm{x}=2.256 r_{\text {exit }} \quad$ (bottom) $\quad \mathrm{x}=11.278 r_{\text {exit }}$ downstream of the nozzle exit.

\subsection{Turbulent Kinetic Energy Profiles}

The normalized turbulent kinetic energy (TKE) along the axis of symmetry at $P_{\text {in }}=140 \mathrm{kPaa}$ and $\mathrm{P}_{\text {in }}=$ $160 \mathrm{kPaa}$ is compared between different turbulence models in Figure 10. The transport of TKE to the jet centerline and the evolution of TKE predicted by the two turbulence model is almost the same. The Realizable $\mathrm{k}-\varepsilon$ model, however, predicts some turbulence upstream and downstream of the nozzle exit for $\mathrm{x} / \mathrm{r}_{\text {exit }} \lesssim 12$. This level of turbulence is absent in the predictions of the SST $\mathrm{k}-\omega$ model, which 
predicts almost zero turbulence along the symmetry axis up to $\mathrm{x} / \mathrm{r}_{\text {exit }} \approx 12$, where the potential core exists.

After the end of the potential core, in the shear layer region, the diffusion of turbulence reaches to its maximum value at a downstream distance of $\mathrm{x} / \mathrm{r}_{\text {exit }} \approx$ 18, where the normalized TKE graph attains a maximum value. This maximum value is slightly greater than 0.04 for the Realizable $\mathrm{k}-\varepsilon$ model and slightly less than 0.04 for the SST $\mathrm{k}-\omega$ model. The dissipation of the TKE after the peak point is slightly higher for the SST $\mathrm{k}-\omega$ model for $\mathrm{x} / \mathrm{r}_{\text {exit }}<22$, and thereafter the Realizable $\mathrm{k}-\varepsilon$ model predicts a slightly faster decay, and consequently more mixing, farther downstream.

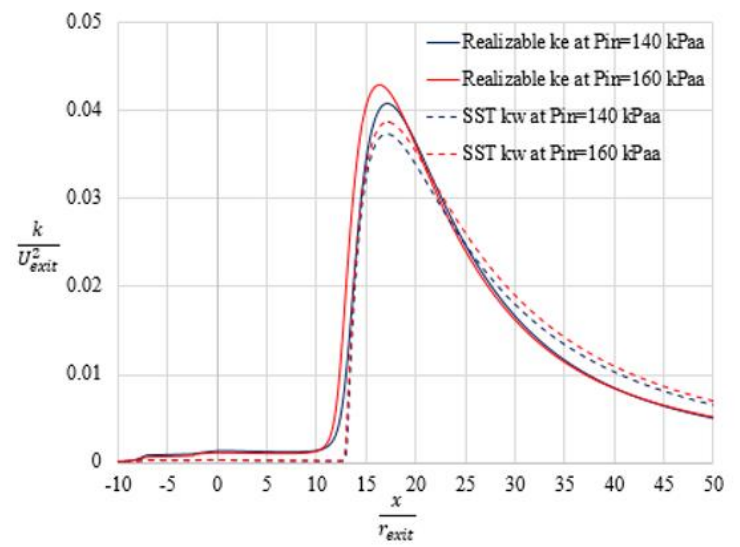

Figure 10: The normalized turbulent kinetic energy along the axis of symmetry predicted by the turbulence models at $P_{\text {in }}=140 \mathrm{kPaa}$ and $P_{\text {in }}=160 \mathrm{kPaa}$.

\subsection{Mach Number Profiles}

Figure 11 shows the Mach number profiles along the symmetry axis predicted by both turbulence models at $P_{\text {in }}=140 \mathrm{kPaa}$ and $P_{\text {in }}=160 \mathrm{kPaa}$. Generally, both turbulence models predict almost the same Mach number evolution inside and outside the nozzle. The location of the shocks in the nozzle (when $P_{\text {in }} \geq$ $160 \mathrm{kPaa}$ ) predicted by the two models are the same. In addition, both models show that the sonic flow condition along the centerline is always reached at the constant throat around $x / r_{\text {exit }}=-3.2$, just upstream of the divergent section (which starts at $\left.x / r_{\text {exit }}=-2.556\right)$.

However, when the flow is subsonic ( $P_{\text {in }} \leq$ $140 k P a a)$, the SST $k-\omega$ model predicts slightly higher flow velocities than the Realizable $k-\varepsilon$ model in the converging and constant-throat sections (between-9.3<x/ $r_{\text {exit }}<-2.6$ ). The difference disappears after that and the profiles perfectly match.

When the flow is supersonic ( $P_{\text {in }} \geq 160 \mathrm{kPaa}$ ), both turbulence models predict the same Mach number profiles inside the nozzle. Outside the nozzle, there are some slightly higher values predicted by the Realizable $\mathrm{k}-\varepsilon$ model within the potential core, i.e, $0 \leq x / r_{\text {exit }} \leq 10$, followed by a slightly earlier decay in velocity. The difference disappears after that and the profiles match. Another difference between the SST $k-\omega$ and Realizable $k-\varepsilon$ models is that the former model predicts a sharp velocity decay; whereas the latter model predicts a much smoother velocity decay at the end of the potential core.

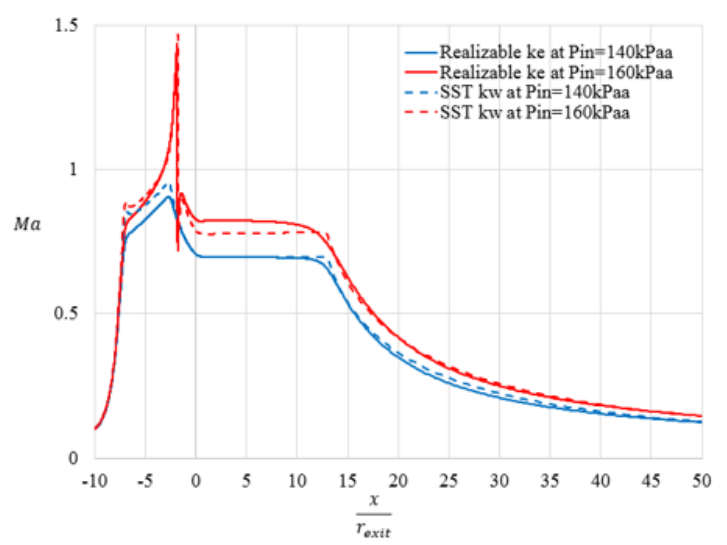

Figure 11: Mach number profile along the jet symmetry axis predicted by different turbulence model at $P_{\text {in }}=140 \mathrm{kPaa}$ and $\mathrm{P}_{\text {in }}=160 \mathrm{kPaa}$.

\subsection{Entrainment Rate Profiles}

This specific nozzle is used to provide gas flares with air to facilitate smokeless combustion. Therefore, it is important to quantify the variation of the mass entrainment along the jet centerline with the upstream pressure. The total mass flow rate through a given cross-section downstream of the nozzle is denoted by $M$ and the mass flow rate at the nozzle exit is denoted by $M_{\text {exit }}$.The entrainment rate coefficient, defined as $K_{e}=\frac{\mathrm{dM}}{\mathrm{dx}} \frac{r_{\text {exit }}}{M_{e}}\left(\frac{\rho_{e}}{\rho_{x}}\right)^{0.5}$ [27], where $\rho_{e}$ and $\rho_{x}$ are the initial and entrained fluid densities, respectively, increases linearly as shown in Figure 12. For sake of illustrations, $K_{e}$ graphs at lower pressure (where nozzle flow is subsonic with $0.5<M a<0.9$ ) and higher pressure $(1.5<M a<2.0)$ are not shown on the graph. However, the normalized entrainment graphs at operating pressures of $120 \mathrm{kPaa} \leq \mathrm{P}_{\text {in }} \leq$ $180 \mathrm{kPaa}$ almost collapse on the presented graphs with insignificant difference. 


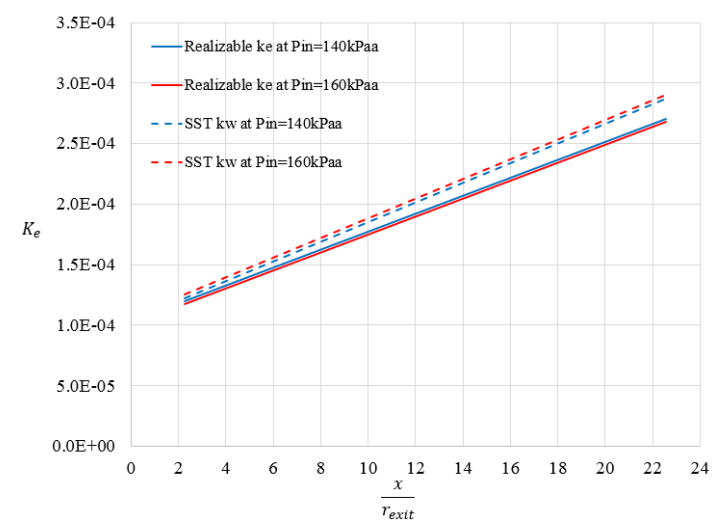

Figure 12: The entrainment rate coefficient $K_{e}$ as a function of distance along the jet for different turbulence models.

\section{CONCLUSIONS}

Numerical studies of compressible flow in a specific converging diverging nozzle were conducted. The nozzle consists of converging and diverging sections connected via a throat section with a finite length and constant diameter. The jet generated by the nozzle is used to control the performance of typical industrial flare systems by entraining the right amount of air into the flame to assure a smokeless flame. The numerical studies characterized the nozzle performance at various pressure ratios (NPR) in the range $1.18 \leq$ NPR $\leq 1.78$ for two different turbulence models. The mean axial velocity profiles at different streamwise locations, mass entrainment rates, the turbulent kinetic energy and Mach number along the jet centerline were presented.

In the case of subsonic nozzle flow, the mean axial velocity profiles were found to be insensitive to the turbulence models used. However, for supersonic flow this insensitivity was seen only downstream of the nozzle exit and outside the potential core region, while within this region the SST $k-\omega$ model showed an unexpected deficit in the mean axial velocity profiles. The turbulent kinetic energy profiles for different turbulence models follow each other closely, with the Realizable $\mathrm{k}-\varepsilon$ model predicting a slightly higher peak value.

For this nozzle design, the flow along the centerline becomes supersonic at the end of the finite-length throat right before entering the diverging part. The turbulence models capture the same normal shock location along the nozzle symmetry axis. The SST k$\omega$ predicts a slightly higher Mach number in the converging and constant-throat parts of the nozzle when the flow is subsonic. The Realizable $\mathrm{k}-\varepsilon$ model, on the other hand, predicts a slightly higher velocity in the potential core when the flow is supersonic. The entrainment rate coefficient increases linearly with the distance along the jet centerline, as reported in [27].

Finally, it is found that the SST $k-\omega$ model, which is a superior model when the flow contains a separation region, predicts an unrealistic velocity deficit in the potential core when the flow became supersonic inside the nozzle. This velocity deficit was not associated with any flow separation at the wall as the diverging section angle in this nozzle was relatively small and no flow separation was observed for the flow.

\section{ACKNOWLEDGEMENTS}

The authors would like to thank Saudi Aramco and NSERC (Natural Sciences and Engineering Research Council of Canada) for their support of this research.

\section{REFERENCES}

[1] M. Mashour, S. Smith, N. Palfreeman, G. Seefeldt, "New Technology: Saudi Aramco High Pressure Air Assist System (HPAAS) for upgrading existing flares to smokeless combustion". International Flame Research Foundation 16th Members Conference, June 2009.

[2] A.H. Shapiro, "The Dynamics and Thermodynamics of Compressible Fluid Flow”, Vol. 1, John Wiley \& Sons, 1953.

[3] D. Anderson, J.D Anderson, "Fundamentals of Aerodynamics", third edition, McGrawHill International Editions, 2001.

[4] J. E. Eggers, "The velocity profiles and eddy viscosity distributions downstream of a Mech 2.22 nozzle exhausting into quiescent air", NASA Technical Note, NASA TN D3601, September 1966.

S.F. Birch, J.E. Eggers, "A critical review of the experimental data for developed free turbulent shear layers", Free Turbulent Shear Flows, NASA SP 321, January 1973.

[6] J.C. Lau, P.J. Morris, M.J. Fisher, "Measurements in subsonic and supersonic free jets using a laser velocimeter", Journal of Fluid Mechanics, Vol. 93, Issue 1, 1-27, 1979. 
[7] J.M. Seiner, M.K. Ponton, B.J. Jansen, N.T. Lagen, "The effects of temperature on supersonic jet noise emission", DGLR/AIAA 14th Aeroacoustic Conference, Aachen, Germany, paper 9202-046, February 1992.

[8] N.J. Georgiadis, J.R. DeBonis. "NavierStokes analysis methods for turbulent jet flows with application to aircraft exhaust nozzles", Progress in Aerospace Sciences, Vol. 42, 377-418, 2006.

[9] L.D. Koch, J.E. Bridges, A. Khavaran, "Flowfield comparisons from three NavierStokes solvers for an axisymmetric separate flow jet”, AIAA Paper 2002-0672, 2002.

[10] S. Sarkar, G. Erlebacher, M.Y. Hossaini, H.O. Engblow, "The analysis and modelling of dilatational terms in compressible turbulence ", Journal of Fluid Mechanics, Vol. 227, 473-493, 1991.

[11] S. Sarkar, "Modeling the pressure-dilatation correlation", NASA Technical Report, May 1991.

[12] N.J, Georgiadis, T.W Aerodynamic Design and Analysis of High Performance Nozzles for Mach 4 Accelerator Vehicles", 44th AIAA Aerospace Sciences Meeting and Exhibit, Reno, Nevada, AIAA paper 490, January 2006.

[13] N.J. Georgiadis, D. A. Yoder, W.A. Engblom, "Evaluation of modified twoequation turbulence models for jet flow predictions", AIAA Journal, Vol. 44, No. 12, December 2006.

[14] Q. Xiao, H.M. Tsai, D. Papamoschou, A. Johnson, "Experimental and numerical study of jet mixing from a shockcontaining nozzle", Journal of Propulsion and Power, Vol. 25, No.3, May-June 2009.

[15] T. DalBello, N. Georgiadis, D. Yoder, T. Keith, "Computational study of axisymmetric off-design nozzle flows", 42nd Aerospace Sciences Meeting and Exhibit, January 2004.

[16] D.S. Tandra, A. Kaliazine, D.E. Cormack and H.N Tran, "Numerical simulation of supersonic jet flow using a modified $k-\varepsilon$ model", International Journal of Computational Fluid Dynamics, Volume 20; 19-27, August 2006.

[17] S. Sarkar, B. Lakshmanan, "Application of a Reynolds Stress turbulence model to the compressible shear layer", AIAA Journal, May 1991.

[18] S. Sarkar, B. Lakshmanan, "Modelling the pressure-strain correlation of turbulence: an invariant dynamical systems approach", Journal of Fluid Mechanics, Vol. 227, 245272, June 1991.

[19] V. Lijo, H.D. Kim, T. Setoguchi, S. Matsuo, "Numerical simulation of transient flows in a rocket propulsion nozzle", International Journal of Heat and Fluid Flow, 31(3):409417, June 2010

[20] A. Balabel, A.M. Hegab, M. Nasr, Samy M. El-Behery, "Assessment of turbulence modeling for gas flow in two-dimensional convergent-divergent rocket nozzle", Applied Mathematical Modelling, January 2011.

[21] D.C. Wilcox, Turbulence Modeling in CFD, DCW Industries, Inc. 1994.

[22] W.C. Reynolds, "Fundamental of turbulence and turbulence modelling and simulation", Lecture notes for Von Karman Institute Agard Report No. 775, 1987.

[23] F. R. Menter, "Two-equation eddy-viscosity turbulence models for engineering applications", AIAA Journal, 32 (8); 15981605, 1994.

[24] P.J. Roache, "Quantification of uncertainty in computational fluid dynamics", Annual Review of Fluid Mechanics, 29; 23-60, 1997.

[25] A.T. Nguyen, H. Deniau, S. Girard, T.A.D. Roquefort, "Unsteadiness of flow separation and end-effects regime in a thrust-optimized contour rocket nozzle", Flow, Turbulence and combustion, 17; 161-181, 2003.

[26] J. Bridges, M. Wernet. "Measurements of the aeroacoustic sound source in hot jets", Tech. Rep. NASA/TM-2004-212508, NASA, Cleveland, OH, February 2004. 
[27] F. Medrano, Y. Fukumoto, C.M. Velte, A. Hodzic, "Mass entrainment rate of an ideal momentum turbulent round jet", Journal of the Physical Society of Japan, Vol. 86, 034401 February 2017. 